\title{
Evaluating the Effect of Health Education Intervention on the Health Beliefs and Behaviors of First-Degree Female Relatives of Breast Cancer Patients
}

\author{
Sule Olgun ${ }^{1}$ Berna Dizer ${ }^{1}$ \\ ${ }^{1}$ Izmir Kavram Medical Services and Techniques Department, Izmir, \\ Turkey
}

South Asian J Cancer 2022;11(1):14-18.
Address for correspondence Sule Olgun, PhD, Izmir Kavram Vocational School, Oguzlar District 1251/2 Street, Number/8, Konak, Izmir, 35320, Turkey (e-mail: sule_olgun1985@hotmail.com).

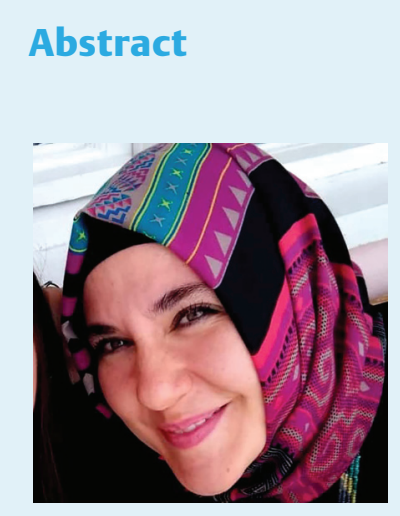

Sule Olgun

Background Breast cancer risk increases by $80 \%$ in the presence of BRCA1 and BRCA2 gene mutations in the same family. In particular, a woman whose sister or mother has breast cancer has a 2- to 5-fold higher risk of developing breast cancer compared with other women. For this reason, recommendations should have been made regarding breast cancer prevention and/or early detection for women with first-degree family history of breast cancer.

Aim The aim of this study was to evaluate the effect of health education, which was provided to first-degree female relatives of breast cancer patients, on their health beliefs and behaviors.

Study Design and Methods The study sample included 50 women with a first-degree relative being treated for breast cancer in the chemotherapy and radiotherapy unit of a university hospital. A one-group pretest-posttest design was used. The pretest consisted of the health belief model scale and a questionnaire regarding the women's sociodemographic information and breast cancer screening behaviors. After the pretest, the patients received health education regarding breast cancer risk factors and screening methods. The posttest was conducted 3 weeks after the education using the same assessment tools.

Keywords

- Breast cancer

Results After education, there were statistically significant increases in rates of practicing breast self-examination, having clinical breast examinations, and undergo-

- cancer

- first-degree relative

- health

- health education ing breast ultrasound/mammography compared with pretest results.

Conclusions Health workers should possess knowledge and experience about breast cancer which will enable them to effectively undertake an educational role, especially for high-risk groups such as women with first-degree family history of breast cancer.

DOI https://doi.org/10.1055/s-0041-1733318 ISSN 2278-330X

How to cite this article: Olgun S, Dizer B. Evaluating the Effect of Health Education Intervention on the Health Beliefs and Behaviors of First-Degree Female Relatives of Breast Cancer Patients South Asian J Cancer 2022;11(1):14-18. (c) 2021. MedIntel Services Pvt Ltd. All rights reserved.

This is an open access article published by Thieme under the terms of the Creative Commons Attribution-NonDerivative-NonCommercial-License, permitting copying and reproduction so long as the original work is given appropriate credit. Contents may not be used for commercial purposes, or adapted, remixed transformed or built upon. (https://creativecommons.org/licenses/by-nc-nd/ 4.0/)

Thieme Medical and Scientific Publishers Pvt. Ltd., A-12, 2nd Floor, Sector 2, Noida-201301 UP, India 


\section{Introduction}

Breast cancer, which is more common in developed countries, causes the death of 2.1 million women every year. As many as 627,000 women died of breast cancer in $2018 .^{1}$ According to 2017 data from the Turkish Ministry of Health, breast cancer ranks first among the 10 most common cancers in women. ${ }^{2}$

Studies of first-degree female relatives of breast cancer patients have described their need for information and support as well as health behaviors. In their study conducted in the US, Sinicrope et al found that daughters of women with breast cancer wanted information about breast self-examination (BSE), clinical breast examination (CBE), mammography, their risk of developing breast cancer, and healthy lifestyle. ${ }^{3}$ Tunin et al reported that prevention was generally an important issue for women who had first-degree relatives with breast cancer. ${ }^{4}$ Aslan determined that the need for information was of greater importance than the need for support among women in Izmir who had a first-degree relative with breast cancer. ${ }^{5}$ Despite the emphasis in the literature on the need for information and support in this group, two studies reported that these women postpone their own health practices while worrying about the health of their relatives, and undergo fewer diagnostic procedures that facilitate early detection compared with other women. ${ }^{6}$

Through health education, health care professionals can influence perceptions of health and disease and encourage preventive behavior modifications. ${ }^{7}$ In this regard, as nurses are constantly interacting with the patients in an informative and supportive role, they are key members of the health care team in terms of providing information about breast cancer risk factors and prevention.

Gaining a better understanding of women who have firstdegree family history of breast cancer and the factors associated with their health beliefs and behaviors may guide the development of breast cancer prevention and screening programs. Previous studies have evaluated this population's need for information and support, women's views on breast cancer and fear of cancer, women's awareness and sensitivity about breast cancer screening, information-seeking behavior of first-degree relatives of women with breast cancer, and the efficacy of health education in eliminating their need for information. ${ }^{5,8}$ However, the effect of health education on the health beliefs of first-degree relatives of women with breast cancer has yet to be determined.

\section{Research Objectives}

\section{Research Objectives 1}

The first-degree female relatives of breast cancer patients should be undergoing breast cancer screening, so they will be presented early with breast cancer symptoms for early detection.

\section{Research Objectives 2}

The education should be provided to first-degree female relatives of women with breast cancer positive affect their health beliefs.

\section{Materials and Methods}

\section{Study Design}

This was a quasi-experimental study performed to determine the effect of education on the health beliefs and behaviors of women with first-degree family history of breast cancer.

\section{Study Setting}

The study was performed in the chemotherapy and radiotherapy unit of a university hospital between September 2014 and April 2015. After obtaining institutional approval, a room in the chemotherapy unit was used to provide health education to the relatives of breast cancer patients.

\section{Study Population and Sample}

The size of the sample was statistically determined by power analysis. As a result of the power analysis, it was found that 50 breast cancer patient relatives who met the research criteria would be sufficient. The study sample included 50 women who had a first-degree relatives being treated for breast cancer in the chemotherapy and radiotherapy unit of a university hospital, could be contacted by the researchers, and met the inclusion criteria. These criteria were being female, having a first-degree relative with breast cancer, having no personal history of cancer, being at least 18 years of age, being literate, having no vision or hearing loss that would prevent communication, being able to communicate and interact with the researcher, being able to attend scheduled appointments, and willingly volunteering to participate in the study.

\section{Data Collection Tools}

Data was collected using the health belief model scale (HBMS) and a personal information questionnaire about the participants.

\section{Ethical Considerations}

Written consent was obtained from The University Health Application and Research Center Clinical Research Ethics Committee (dated August 22, 2014, and numbered 190$55)$, the institution where the research was conducted, and the patients' first relative who participated in the study.

\section{Personal Information Questionnaire}

Based on the literature, the questionnaire comprised 37 items regarding the participants' sociodemographic characteristics. The form also included items evaluating the participants' knowledge of this subject and their breast cancer screening behavior.

\section{Health Belief Model Scale for Breast Cancer Screening Behaviors}

The HBMS was developed by Champion in 1984 based on the HBMS in relation to early breast cancer detection methods. The HBMS was adapted to Turkish in three different studies. In the present study, we used the Turkish version prepared by Gozum and Aydin in 2004. ${ }^{9}$

The HBMS comprises 52 items in eight dimensions that evaluate the individual's opinions about breast cancer and 
general health: susceptibility (3 items), seriousness (6 items), health motivation (5 items), barriers to BSE (8 items), benefits of BSE (4 items), confidence of BSE (10 items), benefits of mammography ( 5 items), and barriers to mammography (11 items). The items are scored using a 5-point Likert-type scale ( $1=$ definitely disagree, $2=$ disagree, $3=$ indecisive, 4 =agree and $5=$ absolutely disagree).

\section{Data Collection Process}

The personal information questionnaire and HBMS were piloted with five first-degree relatives of women with breast cancer. Upon determining there were no problems in understanding the questions, the data collection process was initiated.

Data was collected via face-to-face interviews with firstdegree female relatives of breast cancer patients who presented to the chemotherapy and radiotherapy unit of a university hospital. The pretest-posttest comparison was used in this study. After conducting the pretest, the participants received the education intervention, followed by the posttest. Data collection was completed in approximately 15 minutes. Prior to the study, the participants were informed about the purpose of the study, and those who agreed to participate $(n=50)$ were included in the study after informed consent was obtained.

In the pretest (stage 1), the questionnaire and Champion's breast cancer HBMS were implemented for the first time. About three to four patients' relatives could be contacted per day. The aim was to collect the participants' descriptive information, and determine their breast cancer risk levels, screening behaviors and knowledge levels before the education.

In the education intervention (stage 2 ), the participants were informed about breast cancer and relevant risk factors, prevention methods, and screening behaviors in face-to-face sessions. At the end of the session, the participants were provided a booklet containing the information for future reference. This stage occurred approximately 3 weeks after the pretest. The aim was to answer the participants' questions about breast cancer, risk factors and prevention of breast cancer, and breast cancer screening behavior, with a particular focus on correcting misinformation.

Each education session included four to six participants and lasted 40 to 50 minutes, depending on the questions asked by the attendees. A 10-minute break was given every 30 minutes if necessary. About 3 weeks after the education (stage 3), the participants completed the personal information questionnaire and HBMS a second time. The aim was to measure how effective the educational intervention was and to what degree it altered the participants' behavior (i.e., determine its effect on their health beliefs).

During the study, the participants were told they could contact the researcher regarding any topic and were provided a contact phone number. Each meeting date was approximately 3 weeks later. The time between intervention and posttest was no less than 3 weeks and no more than 4 weeks. Therefore, there was an interval of approximately 6 weeks between the pretest and posttest questionnaire and HBMS.

\section{Development of the Education Material}

The health education intervention provided participants with information on breast cancer, prevention methods, risk factors, and early detection. After the intervention, the participants were given an informative booklet compiled by the researcher.

After the booklet was prepared, it was shown to two women from different educational backgrounds who both had first-degree relatives with breast cancer, and feedback was obtained on its understandability. The booklet contained information about the structure of the breast, description and symptoms of breast cancer, breast cancer risk factors, prevention methods, and screening methods used for the early detection of breast cancer.

\section{Data Analysis}

Statistical Package for the Social Sciences (SPSS) v15.0 computer software was used to analyze the study data. Alpha levels of 0.05 and 0.001 were used to evaluate the results of statistical analyses. $p$ values at or below these levels were

Table 1 Analysis of breast cancer screening behavior before and after health education intervention $(n=50)$

\begin{tabular}{|c|c|c|c|c|c|c|c|c|c|}
\hline \multicolumn{2}{|c|}{ Breast cancer screening behaviors } & \multicolumn{5}{|c|}{ Postintervention } & & & \multirow{3}{*}{$\begin{array}{l}\text { McNemar } \\
p\end{array}$} \\
\hline \multirow[t]{4}{*}{ BSE } & & & Yes & & No & & Tot & & \\
\hline & & & $n$ & $\%$ & $n$ & $\%$ & $n$ & $\%$ & \\
\hline & \multirow[t]{2}{*}{ Preintervention } & Yes & 27 & 54.0 & 0 & 0.0 & 27 & 54.0 & \multirow[t]{3}{*}{0.000} \\
\hline & & No & 16 & 32.0 & 7 & 14.0 & 23 & 46.0 & \\
\hline Total & & & 43 & 86.0 & 7 & 14.0 & 50 & 100.0 & \\
\hline \multirow[t]{2}{*}{ CBE } & \multirow[t]{2}{*}{ Preintervention } & Yes & 20 & 40.0 & 0 & 0.0 & 20 & 40.0 & \multirow[t]{3}{*}{0.000} \\
\hline & & No & 16 & 32.0 & 14 & 28.0 & 30 & 60.0 & \\
\hline Total & & & 36 & 72.0 & 14 & 28.0 & 50 & 100.0 & \\
\hline \multirow[t]{2}{*}{ Ultrasound/mammography } & \multirow[t]{2}{*}{ Preintervention } & Yes & 27 & 54.0 & 1 & 2.0 & 28 & 56.0 & \multirow[t]{3}{*}{0.002} \\
\hline & & No & 13 & 26.0 & 9 & 18.0 & 22 & 44.0 & \\
\hline Total & & & 40 & 80.0 & 10 & 20.0 & 50 & 100.0 & \\
\hline
\end{tabular}

Abbreviations: BSE, breast self-examination; CBE, clinical breast examination. 
considered statistically significant and highly significant, respectively. Pretest to posttest comparisons were made using McNemar test for repeated measures related to breast cancer screening behaviors, and paired samples $t$-test for mean HBMS subscale scores.

\section{Results}

From pre- to postintervention, the proportion of women who performed BSE increased from $54 \%(n=27)$ to $86 \%$ $(n=43)$, those who underwent clinical breast examination increased from $40 \%(n=20)$ to $72 \%(n=36)$, and those who had ultrasound/mammography increased from $56 \%(n=28)$ to $80 \%(n=40)$, all of which were statistically significant according to McNemar analysis $(p<0.01)$ ( - Table 1 ).

There were no significant pre- to postintervention changes in mean susceptibility ( $t=0.897, p=0.374)$, seriousness $(\mathrm{t}=1.565, p=0.124)$, or health motivation $(\mathrm{t}=-1.132$, $p=0.263)$ subscale scores $(p>0.05)$. However, significant changes were detected in postintervention mean HBMS scores for benefits of BSE ( $t=-2.878, p=0.006)$, barriers to BSE $(\mathrm{t}=3.203, p=0.002)$, confidence in BSE $(\mathrm{t}=-11.493$, $p=0.000)$, benefits of mammography $(t=-2.956, p=0.005)$, and barriers to mammography ( $\mathrm{t}=3.788, p=0.000)$ subscales $(p<0.01)$ ( - Table 2).

It was determined that $52 \%$ of women had breast ultrasound before training, which increased to $80 \%$ after training, and the difference between the values before and after the training was statistically significant $(p<0.05)$. It was determined that the rates of women having mammography before and after the training did not change (40\%) and the difference between the values before and after the training was not statistically significant $(p>0.05)$. It was determined that $48 \%$ of women included in the sample group had breast ultrasound for control purposes before the training, and the rate of breast ultrasound for control purposes increased to $76 \%$ after the training.

\section{Discussion}

In the present study, the mean HBMS score for perceived benefits of BSE was $15.44 \pm 3.16$ before the education intervention, and increased to $18.00 \pm 5.78$ after the intervention. This increase was highly statistically significant $(p<0.01)$ ( - Table 1$)$. The increase in the HBMS perceived benefits of BSE indicates that the women had a better understanding of the importance of practicing BSE after the education.

Before the intervention in our study, the mean HBMS score on the perceived barriers to BSE subscale was $18.82 \pm$ 5.04 , and it decreased significantly to $16.20 \pm 5.01$ after the intervention $(p<0.01)$ ( - Table 2).

Factors identified in the literature as barriers to BSE include insufficient time, knowledge/skill, or self-confidence to perform BSE; doubt regarding the effectiveness of BSE; fear of breast cancer; forgetfulness; and feeling that BSE is uncomfortable or difficult. ${ }^{9}$ We believe that the reduction in perceived barriers to BSE observed in our study was achieved by identifying the women's barriers to BSE and providing appropriate education that supplied the information they needed, corrected their misconceptions about BSE, and ensured that they understood the importance of BSE. This demonstrates the positive effect of the health education we provided to these women on their health beliefs. Our findings suggest that if nurses determine the factors acting as barriers to BSE, plan education interventions to eliminate these factors, and repeat these interventions frequently at regular intervals, the perceived barriers to BSE among women can be effectively reduced.

According to the HBMS, reported barriers to mammography include factors such as mammography being a painful, problematic, and time-consuming procedure; the rude behavior of staff working in this unit toward women; fear of radiation exposure; forgetting to make an appointment; fear of cancer; not knowing where to go for mammography; mammography being performed after a certain age; and women's perception of having more important problems. In addition, many women do not consider mammography a must, and the high cost of the procedure, its unavailability in some centers, and the belief that radiation exposure from mammography is itself a risk factor for breast cancer may also deter women from undergoing mammography.

Before the educational intervention, the women in our study stated that they had prejudices and concerns about

Table 2 Analysis of mean HBMS for breast cancer subscale scores before and after health education intervention $(n=50)$

\begin{tabular}{|l|l|l|l|l|l|l|l|}
\hline & \multicolumn{2}{|l}{ Preintervention } & \multicolumn{2}{l|}{ Postintervention } & \\
\hline HBMS Subdimensions & Min & Max & Mean \pm SD & Min & Max & Mean \pm SD & t/p \\
\hline Susceptibility & 3 & 14 & $8.16 \pm 2.48$ & 6 & 12 & $8.54 \pm 2.65$ & $0.897 / 0.374$ \\
\hline Seriousness & 11 & 30 & $19.22 \pm 5.66$ & 12 & 30 & $17.82 \pm 5.82$ & $1.565 / 0.124$ \\
\hline Health Motivation & 5 & 25 & $20.54 \pm 3.86$ & 15 & 25 & $21.20 \pm 2.40$ & $-1.132 / 0.263$ \\
\hline Benefits of BSE & 4 & 20 & $15.44 \pm 3.16$ & 16 & 56 & $18.00 \pm 5.78$ & $-2.878 / 0.006$ \\
\hline Barriers to BSE & 8 & 30 & $18.82 \pm 5.04$ & 8 & 32 & $16.20 \pm 5.01$ & $3.203 / 0.002$ \\
\hline Confidence in BSE & 20 & 49 & $30.80 \pm 7.89$ & 38 & 50 & $44.34 \pm 5.07$ & $-11.493 / 0.000$ \\
\hline Benefits of mammography & 12 & 25 & $17.68 \pm 2.86$ & 5 & 25 & $19.54 \pm 4.33$ & $-2.956 / 0.005$ \\
\hline Barriers to mammography & 11 & 44 & $28.44 \pm 6.95$ & 11 & 52 & $23.38 \pm 6.71$ & $3.788 / 0.000$ \\
\hline
\end{tabular}

Abbreviations: BSE, breast self-examination; HBMS, health belief model scale. 
mammography, that they did not have sufficient information about mammography, and that their health beliefs on this issue were shaped by the experiences of others. However, their mean HBMS perceived barriers to mammography subscale scores were $28.44 \pm 6.95$ before the education and decreased significantly to $23.38 \pm 6.71$ after the education $(p<0.01)$ ( $>$ Table 2 ). ${ }^{3-10}$ The decrease in HBMS barriers to mammography subscale score after the intervention suggests that it eliminated the gaps in their knowledge about mammography and dispelled their prejudices against the procedure.

After the training intervention, the rate of women who had ultrasonography and mammography increased. This result is thought to be a result of the positive effect of health education on women's health beliefs. Women who have high perceived benefits and low perceived barriers to mammography according to HBMS are expected to have a more positive view of having mammography in the future. It is thought that if nurses educate women about the importance of mammography in the early diagnosis of breast cancer and correct their misinformation, prejudices against mammography may decrease.

\section{Conclusion}

It is thought that mammography, which women do not need, is an expensive screening method. It is not in every hospital and the radiation during mammography is seen as a breast cancer risk factor, causing them to avoid mammography. In addition, it is thought that while women worry about the health of their relatives with breast cancer, they also have difficulty in adapting to early diagnosis practices. It is considered that nurses play a great role in planning research and training programs for breast health to understand and develop health beliefs that are effective in women's BSE, clinical breast examination, ultrasound and mammography.

This study showed that a health education intervention about breast cancer resulted in significant increases in rates of performing BSE, undergoing $\mathrm{CBE}$, and having breast ultrasonography/mammography as well as significant changes in HBMS scores for benefits of BSE, barriers of BSE, confidence in BSE, benefits of mammography, and barriers of mammography. In light of these results, we recommend clarifying the roles of breast care nurses, who assist patients and their relatives in the process of choosing optimal therapy, and increasing the number of breast care nursing certification programs. We also believe that further research is needed to investigate the need for information and support among women with firstdegree family history of breast cancer in Turkey and evaluate long-term outcomes in trials of different education models.

The perception of the disease as a threat is different between the individuals who have a family history of cancer and develop cancer cases and the individuals who do not have cancer cases. Individuals with cancer cases in their family may perceive this disease as a high threat to themselves. For this reason, we think that they may have changed their behavior with our training.

\section{Study Limitations}

Reaching first-degree female relatives of our breast cancer patients was difficult because most came to the hospital with their male spouses. In addition, some of the women recruited for the study did not want to leave the patients alone during their treatment, and either wanted the training to be shorter or did not want to complete the posttest evaluation and withdrew from the study after the educational intervention. This prolonged the data collection process. Moreover, as the posttest was held approximately 3 weeks after the intervention, we cannot evaluate the long-term effects of the education.

\section{Ethics Committee Approval}

Ethics committee approval was received for this study from The University Health Application and Research Center Clinical Research Ethics Committee (dated 22.08.2014 and numbered 190-55).

\section{Funding}

The authors declared that this study has received no financial support.

\section{Conflict of Interest}

There are no conflicts of interest.

\section{References}

1 Tunin R, Uziely B, Woloski-Wruble AC. First degree relatives of women with breast cancer: who's providing information and support and who'd they prefer. Psychooncology 2010;19(04): 423-430

2 Word Health Organization. Incidence of Breast Cancer. Accessed June 29, 2021 at: https://www.who.int/cancer/prevention/diagnosisscreening/breastcancer/en/28.10.2019

3 Health Statistics Yearbook. Accessed June 29, 2021 at: http://sbu. saglik.gov.tr/

4 Bragg Leight S, Deiriggi P, Hursh D, Miller D, Leight V. The effect of structured training on breast self-examination search behaviors as measured using biomedical instrumentation. Nurs Res 2000;49 (05):283-289

5 Sinicrope PS, Patten CA, Clark LP, et al. Adult daughters' reports of breast cancer risk reduction and early detection advice received from their mothers: an exploratory study. Psychooncology 2009; 18(02):169-178

6 Aslan G. Determination of Information and Support Needs of First Degree Relatives of Breast Cancer İndividuals [thesis]. Turkey: Ege University, Institute of Health Sciences, Master Thesis; 2006

7 Gozum S, Aydin I. Validity of the Turkish version of Champion's Health Belief Model Scale. Paper presented at: 2nd International 9th National Nursing Congress Book; 2003; Antalya

8 Andic S. Determination of İnformation and Support Needs of Women with First Degree Relatives with Breast Cancer [thesis]. Turkey: Dokuz Eylül University Institute of Health Sciences; 2011

9 Sadıkoglu G. Approach to breast cancer patient in primary care. J Breast Health 2012;3:100-125

10 Ministry of Health, Department of Cancer Control Accessed June 29, 2021 at: http://kanser.gov.tr/kanser/kanser-turleri/49meme-kanseri.html 